\title{
Search for Gamma-ray Line emission from Dark Matter annihilation in the Galactic Centre with the MAGIC telescopes
}

\section{Tomohiro Inada, ${ }^{a, *}$ Daniel Kerszberg, ${ }^{b}$ Moritz Hütten, ${ }^{c}$ Masahiro Teshima, ${ }^{a, c}$ Javier} Rico $^{b}$ and Daniele Ninci ${ }^{b}$ on behalf of the MAGIC Collaboration

\author{
${ }^{b}$ Institut de Física d'Altes Energies (IFAE), \\ 08193 Bellaterra (Barcelona), Spain \\ ${ }^{c}$ Max-Planck-Institut für Physik \\ Föhringer Ring 6, 80805 München, Germany
}

${ }^{a}$ Institute for Cosmic Ray Research, The University of Tokyo

5-1-5, Kashiwa-no-ha, Kashiwa, Chiba 277-8582, Japan

The Barcelona Institute of Science and Technology CampusUAB

E-mail: tomohiro@icrr.u-tokyo.ac.jp

\begin{abstract}
We present the first search for dark matter (DM) spectral lines in the Galactic centre (GC) region with the MAGIC telescopes. The MAGIC telescopes, located on the Canary island of La Palma (Spain), are sensitive to gamma rays in the energy range from $50 \mathrm{GeV}$ to $50 \mathrm{TeV}$. MAGIC has performed indirect DM searches in various astrophysical targets, such as dwarf spheroidal galaxies and clusters of galaxies. Observations at high zenith angles significantly increase the telescopes' collection area and sensitivity for gamma rays in the $\mathrm{TeV}$ regime. We present the results obtained with more than 200 hours of high-zenith angle observations of the GC region with MAGIC, which allow us to probe promising heavy SUSY models, and to obtain competitive limits to the DM annihilation cross-section at high DM particle mass, compared to existing constraints. We will discuss how we exploit the data from a complex sky region to search for a line-like DM signature.
\end{abstract}

$37^{\text {th }}$ International Cosmic Ray Conference (ICRC 2021)

July 12th - 23rd, 2021

Online - Berlin, Germany

\footnotetext{
${ }^{*}$ Presenter

${ }^{\dagger}$ a complete list of the MAGIC Collaboration authors can be found at the end of the proceedings
} 


\section{Introduction}

The recent cosmological precision measurement by Planck [1] supports the existence of nonbaryonic Dark Matter (DM). The $\Lambda$-CDM model presumes DM to be "cold", i.e., non-relativistic at freeze-out, to explain the formation of large-scale structures. One of the most promising candidates for DM are Weakly Interacting Massive Particles (WIMPs), which are widely expected as new fundamental particles at $\mathrm{GeV}-\mathrm{TeV}$ energies in theories beyond the Standard Model of Particle Physics. Various experiments with colliders, underground facilities, and telescopes have been conducted to elucidate the identity and properties of particle DM, but no evidence for non-gravitational DM has been found so far.

Indirect DM searches aim at detecting signatures from particle DM in cosmic messenger particles and can be pursued with gamma-ray telescopes sensitive at very high energies (VHE). In this proceeding, we report the search for line-like gamma-ray signals from DM annihilation in the Galactic Centre (GC) with the Major Atmospheric Gamma Imaging Cherenkov telescopes (MAGIC). Indirect DM searches with gamma rays seek for high-energetic photons produced when DM particles with mass at the electroweak scale $(\mathrm{GeV}-\mathrm{TeV})$ annihilate with each other. Gamma rays are not affected by magnetic fields in the Universe. Therefore, they can be directly related to the production site, allowing to trace the DM abundance and distribution throughout the Universe, with the expected gamma-ray flux from DM annihilation being proportional to the square density of DM along the line of sight. Also, the gamma-ray energy spectrum from DM annihilation has unique spectral features. By reconstructing the energies of these gamma rays, one is potentially able to identify the DM particle physics properties, as e.g., its mass, annihilation cross-section, branching channels, or lifetime [2]. Among the many spectral features related to annihilation of cold DM, the most outstanding one is the emission corresponding to the DM annihilation into two photons, which makes a sharp peak at the DM mass. Such mono-energetic gamma rays at very-high gamma-ray energies (VHE, $>100 \mathrm{TeV}$ ) are a smoking gun to search for DM since there is no astrophysical process with similar signature. However, DM annihilation into two photons is highly suppressed compared to other channels as, e.g., $\mathrm{W}^{ \pm}, \mathrm{Z}$ bosons, quarks, or leptons. Still, several candidates for the Lightest SUSY Particle (LSP) in supersymmetric (SUSY) extensions of the Standard Model of Particle Physics, and being excellent DM candidates, are expected to have an enhanced annihilation cross-section into gamma rays [3]. Therefore, searches for line-like spectral emission from DM annihilation in the VHE regime are a promising strategy. Depending on several parameters, the expected $\gamma$-ray flux $\Phi_{\gamma}$ from DM annihilation can be written as:

$$
\frac{d \Phi_{\gamma}}{d E}=\frac{1}{4 \pi} \frac{\langle\sigma v\rangle}{2 m_{D M}^{2}} \times \frac{d N_{\gamma}}{d E} \times J(\Delta \Omega),
$$

where

$$
\frac{d N_{\gamma}}{d E}=2 \delta\left(E-m_{D M}\right) \quad \text { for annihilation into } \gamma \gamma
$$

and

$$
J(\Delta \Omega)=\int_{\Delta \Omega} d \Omega^{\prime} \int_{\text {l.o.s }} \rho^{2}\left(l, \Omega^{\prime}\right) d l .
$$

Here, $\langle\sigma v\rangle$ is the thermally averaged DM annihilation cross section for a pure $\gamma \gamma$ channel, $m_{D M}$ is the WIMP mass, and $d N_{\gamma} / d E$ is the $\gamma$-ray yield per annihilating into two photons directly. $J(\Delta \Omega)$, 

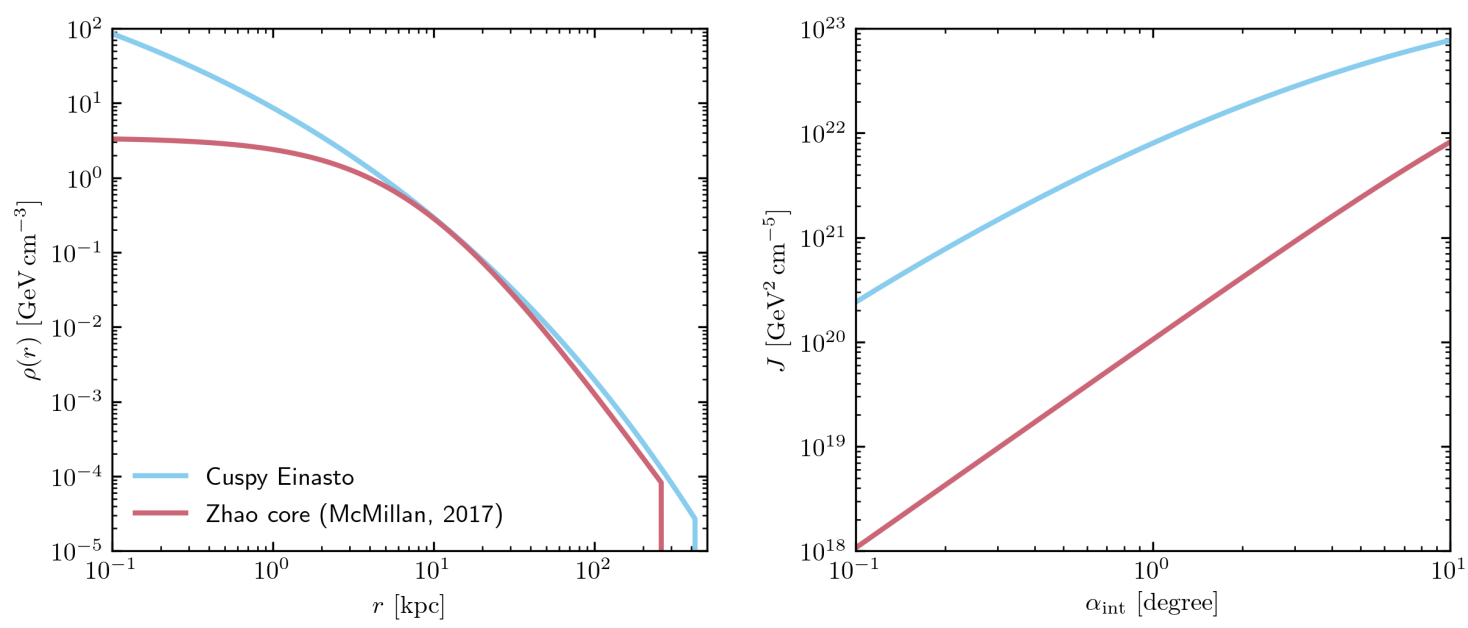

Figure 1: Comparison of the two density models (left) and corresponding $J$-factors (right) as a function of the spatial (left) and angular (right) distance from the GC considered in this work.

the so-called $J$-factor, is the integral of the squared DM density $\rho$ over a solid angle $\Delta \Omega$ and along the observed line of sight (l.o.s). It is expected that out of all possible astrophysical targets for indirect DM searches, the GC provides the largest $J$-factor, due to its dense DM content in close proximity $(\sim 8 \mathrm{kpc})$. From this point of view, the GC is by far the best target for indirect DM searches. On the other hand, the DM density profile in the central few kpc of the Milky Way is not well constrained neither form the theoretical, nor from the observational point of view, due to the difficulties in obtaining kinematic data and a robust prediction about the effect of high baryonic matter density on the DM distribution in the inner Galaxy. Therefore, we scrutinize two different DM density models, a cuspy and a core profile as shown in Fig. 1. The cuspy Einasto profile in Fig. 1 is described by Eq. (4) below [4, 5], which shows a steep density increase towards the inner halo. Here, $r$ is the distance from the center of the profile, $r_{s}$ is the scale radius for a curvature of a profile, and $\rho_{s}$ is the density normalization parameter, and $\alpha$ is the parameter for shape. On the other hand, the Zhao core profile [6] in Fig. 1 assumes a flat core towards the GC, described by Eq. (5) with $(\alpha, \beta, \gamma)=(1,3,0)$ :

$$
\begin{gathered}
\rho_{\text {Einasto }}(r)=\rho_{s} \exp \left\{\frac{-2}{\alpha}\left[\left(\frac{r}{r_{s}}\right)^{\alpha}-1\right]\right\} \\
\rho_{\text {Zhao }}(r)=\frac{2^{\frac{\beta-\gamma}{\alpha}} \rho_{s}}{\left(\frac{r}{r_{s}}\right)^{\gamma}\left[1+\left(\frac{r}{r_{s}}\right)^{\alpha}\right]^{\frac{\beta-\gamma}{\alpha}}}
\end{gathered}
$$

\section{MAGIC observations of the Galactic Centre}

The MAGIC telescopes consist of two 17 m diameter Imaging Atmospheric Cherenkov Telescopes (IACTs) located at the Roque de los Muchachos observatory $\left(28^{\circ} \mathrm{N}, 18^{\circ} \mathrm{W}\right)$ at an altitude of 


\begin{tabular}{lccc}
\hline \hline Profile name & $J\left(0.5^{\circ}\right)$ & $J\left(1.0^{\circ}\right)$ & $J\left(1.1^{\circ}\right)$ \\
\hline Cuspy Einasto & $3.14 \times 10^{21}$ & $8.01 \times 10^{21}$ & $9.03 \times 10^{21}$ \\
Zhao $\gamma=0$ core & $2.66 \times 10^{19}$ & $1.06 \times 10^{20}$ & $1.28 \times 10^{20}$ \\
\hline
\end{tabular}

Table 1: $J$-factor values integrated for our circular ROI around the direction towards the GC and models from [5] and [6]. All values are given in units of $\mathrm{GeV}^{2} \mathrm{~cm}^{-5}$.

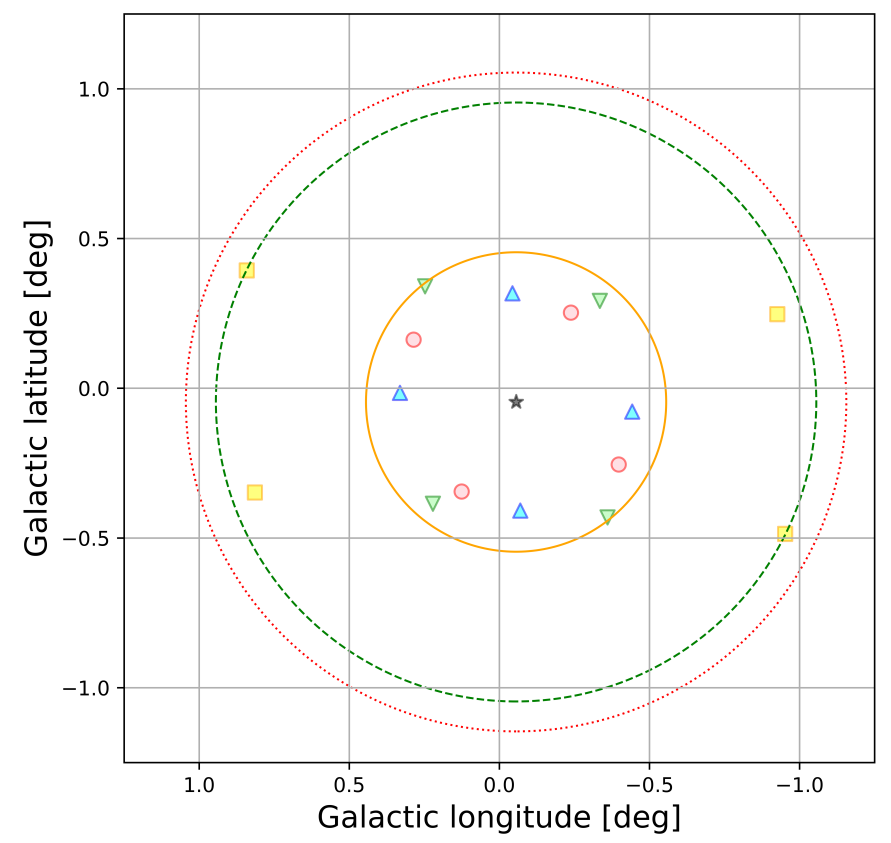

ROI (1.0 deg Offset)

ROI (0.5 deg Offset)

ROI (0.4 deg Offset)

2013, 2014

2015, 2016

2017a, 2018a, 2019

$2017 b, 2018 b$

Figure 2: Each dot shows the pointing directions which were used in different observational periods. Each circle shows the corresponding ROI around the Galactic Center. The red dotted-line shows a $1.1^{\circ}$ radius ROI for the data in 2013 and 2014 (red circles) and blue triangles for the data in (2015 and 2016). The green dashed-dotted line shows a $1.0^{\circ}$ radius ROI for the data in 2017, 2018 and 2019; and the yellow solid-line shows a $0.5^{\circ}$ radius $\mathrm{ROI}$ for the data in 2017 and 2018 .

$2200 \mathrm{~m}$ a.s.l. on the Canary island of La Palma, Spain. The MAGIC telescopes are sensitive to gamma rays from $50 \mathrm{GeV}$ to $50 \mathrm{TeV}$ with observations at low zenith distance [7]. In observations at larger zenith distance, the collection area for gamma rays is significantly boosted at $\mathrm{TeV}$ energies. At the same time, the Cherenkov light is absorbed more strongly by the atmosphere because the distance, and correspondingly, the thickness of the atmosphere, of the particle cascades to the telescopes is increased. This increases the energy threshold and degrades the energy resolution by a few percent. Since the MAGIC telescopes are located in the northern hemisphere, the GC (i.e., $\mathrm{SgrA}^{*}$ ) is visible at a zenith angle $\mathrm{zd}>58^{\circ}$. The GC is an observational target for multiple science purposes [8]. The MAGIC telescopes have observed the GC for six years from 2013 to 2019 in "wobble" observation mode, where the targeted source is observed not in the camera center, but at some angular offset. MAGIC has performed several pointing directions and wobble offsets as shown in Fig. 2. 


\section{Data Analysis}

The data was processed using the MAGIC Analysis and Reconstruction Software (MARS) with the standard MARS analysis chain [9] and applied quality cuts. Also, we used events inside several circular regions of interest (ROI) in the sky as shown in Fig. 2, which were defined depending on the different wobble pointing directions in each observational period. In order to reduce a systematic uncertainty from the camera response (acceptance) close to the camera border, we required each ROI to be within $1.5^{\circ}$ from the camera center. We aim at detecting a sharp spectral line emission from DM annihilation directly into two photons. The expected signal shows a clear peak at the corresponding DM mass. In this work, we use the "sliding window technique" to search for such gamma-ray peaks [10]. We modeled the background with a smooth power-law function inside a given energy window. With this assumption, we perform an interpolation to the background energy spectra to obtain a background model, and searched for a deviation. The loss of sensitivity is less because our background-control region is free of signal events and also we do not need to perform Off region subtraction as for conventional strategies relying on spatial On/Off regions. We use 204 hours of data to perform the analysis to search for a DM line signal. The analysis has been performed with the full likelihood approach $[11,12]$ and using the sliding window technique. The likelihood formula is:

$$
\begin{aligned}
\mathcal{L}_{i}\left(g_{i} ; v_{i} \mid \mathcal{D}_{i}\right) & =\mathcal{L}_{i}\left(g_{i} ; b_{i}, \tau_{i} \mid\left\{E_{j}^{\prime}\right\}_{\left.j=1, \ldots, N_{\mathrm{ON}, i}, N_{\mathrm{ON}, i}\right)}\right. \\
& =\frac{\left(g_{i}+\tau_{i} b_{i}\right)^{N_{\mathrm{ON}, i}}}{N_{\mathrm{ON}, i} !} e^{-\left(g_{i}+\tau_{i} b_{i}\right)} \times \frac{1}{g_{i}+\tau_{i} b_{i}} \prod_{j=1}^{N_{\mathrm{ON}}}\left(g_{i} f_{g}\left(E_{j}^{\prime}\right)+\tau_{i} b_{i} f_{b}\left(E_{j}^{\prime}\right)\right) \\
& \times \mathcal{T}\left(\tau_{i} \mid \tau_{\mathrm{obs}, i}, \sigma_{\tau, i}\right) .
\end{aligned}
$$

where the index $i$ runs over the number of analysis periods composing our data set on the GC. The parameter $v_{i}$ denotes the nuisance parameters, $\mathcal{D}_{i}$ represents the data set, each $g_{i}, b_{i}, N_{\mathrm{ON}, i}$ are the estimated number of signal and background events, and the number of observed events, respectively, in a given energy energy window and ROI. $f_{g}\left(E_{j}^{\prime}\right)$ is the probability density function (PDF) of the signal and $f_{b}\left(E_{j}^{\prime}\right)$ is the one of the background, both as a function of the reconstructed gamma-ray energy of each event, respectively. $f_{g}\left(E_{j}^{\prime}\right)$ is computed as the convolution with the delta-function and the migration matrix for the energy estimator as following $f_{g}\left(E_{j}^{\prime}\right)=2 \delta\left(E-m_{D M}\right) G\left(E^{\prime} \mid E\right)$ where $E$ and $E^{\prime}$ are the true and reconstructed gamma-ray energy, and $G$ is the the migration matrix for the energy estimator. $f_{b}\left(E_{j}^{\prime}\right)$ is the background model, which is modelled by an interpolation of spectra inside an energy window. $\mathcal{T}$ is the likelihood for $\tau_{i}$ (the normalization factor for background base line), parameterized by a Gaussian function with mean $\tau_{\mathrm{obs}, i}$ and variance $\sigma_{\tau, i}^{2}$. The systematic uncertainty in $\tau_{i}$, which is denoted as $\sigma_{\text {syst }}$ and is determined with background data, is considered in our likelihood as an additional contribution to the uncertainty of $\tau$. It is therefore estimated using the equation $\sigma_{\tau}^{2}=\sigma_{\tau \text {,stat }}^{2}+\tau^{2} \cdot \sigma_{\text {syst }}^{2}$, where $\sigma_{\tau \text {,stat }}$ is the statistical error on $\tau . \sigma_{\text {syst }}$ is introduced in the likelihood Eq. (6) as the standard deviation of the Gaussian likelihood term, considering the contribution of a systematic uncertainty of $\tau$. The estimated number of signal events $g_{i}$ depends on the parameter $\langle\sigma v\rangle$ as follows 


$$
g_{i}(\langle\sigma v\rangle)=T_{\mathrm{obs}, \mathrm{i}} \int_{0}^{\infty} d E \frac{d \Phi(\langle\sigma v\rangle)}{d E} \times A_{\mathrm{eff}}(E),
$$

where $\mathrm{T}_{\mathrm{obs}}$ is the observation time in each sample $i . \mathrm{A}_{\mathrm{eff}}$ is the effective collection area. Although $\mathrm{A}_{\mathrm{eff}}$ is computed with gamma-ray MC data, we took into account a spatial distribution of each dark matter profile model for the effective collection area computation, which is called "Donut MC" [13]. Then, we defined a test statistic with the profile likelihood ratio $\lambda_{P}$ in Eq. (8) as

$$
\mathrm{TS}=-2 \ln \lambda_{P}(\langle\sigma v\rangle \mid \mathcal{D})=-2 \ln \left(\frac{\mathcal{L}(\langle\sigma v\rangle ; \hat{\hat{v}} \mid \mathcal{D})}{\mathcal{L}(\langle\widehat{\sigma v}\rangle ; \hat{v} \mid \mathcal{D})}\right),
$$

where $\langle\sigma v\rangle$ is the parameter of interest, and $v$ denotes the nuisance parameters. In the numerator, $\hat{\hat{v}}$ are the nuisance parameter values when the likelihood function $L$ is maximized for a given $\langle\sigma v\rangle$. In the denominator, $\langle\widehat{\sigma v}\rangle$ and $\hat{v}$ are the values maximizing the likelihood function $L$. In absence of any hint for a signal, we set upper limits on $\langle\sigma v\rangle$ at the 1-sided $95 \%$ confidence level (CL) by solving the equation TS $=2.71$. Significant contributions by a systematical offset of the background can bias the estimation of a line-like excess. We estimated possible contaminations of the background determination using data on Non-DM aimed sources. This corresponds to check the uncertainty in the background normalization denoted with $\mathcal{T}$ in Eq. (6) [14]. If the background is perfectly determined, $\tau_{i}$ is equal to 1 . However, the background is not perfectly determined in practice. We applied the same quality cuts and likelihood analysis to the background data (null hypothesis), and evaluated the quantity $R_{\mathrm{j}}^{k}=\mathrm{N}_{\mathrm{ex}, \mathrm{j}}^{\mathrm{k}} / \mathrm{N}_{\mathrm{ON}, \mathrm{j}}^{\mathrm{k}}$, including the statistical fluctuation and potential systematic offsets. Here, the index $j=1, \ldots, 120$ runs over the number of background samples and the index $k=1,2,3$ parametrizes a check of some energy dependence: namely, we divided the test samples in three energy categories, low $(E<3 \mathrm{TeV})$, middle $(3 \mathrm{TeV} \leq E<10 \mathrm{TeV})$ and high $(E \geqq 10 \mathrm{TeV})$ energies. $\mathrm{N}_{\mathrm{ex}, \mathrm{j}}$ is the number of excess events judged as the line component by the likelihood, and $\mathrm{N}_{\mathrm{ON}, \mathrm{j}}$ is the number of events in an energy window according to Eq. (6). If $R$ is zero, it means that the likelihood equation can perfectly distinguish between signal and background events. In fact, $R$ is distributed with a variance of $\sigma_{R}^{2}=\sigma_{\text {stat }}^{2}+\sigma_{\text {syst }}^{2}$ where $\sigma_{\text {stat }}$ is the standard deviation of the $R_{\text {stat }}$, which is the distribution of the statistical uncertainty propagated from each $R_{j}^{k}$. Finally, $\sigma_{\text {syst }}$ is estimated as less than $1 \%$. In order to represent a bias in a background determination, $\tau_{\mathrm{obs}, \mathrm{i}}=1$ $R_{\text {mean }}$ (the mean of the distribution of $R_{j}^{k}$ ) is used.

\section{Results and discussion}

The analysis shows no evidence of a significant line-like gamma-ray excess in the region of $\sim 1^{\circ}$ around the GC and results in competitive upper limits on $\langle\sigma v\rangle$ at the $95 \%$ C.L. for $15 \mathrm{DM}$ masses from $912 \mathrm{GeV}$ to $43 \mathrm{TeV}$ with each of the Einasto and a core profile of the inner Galactic DM density, respectively. The left panel of Fig. 3 shows the consistency of the data with the null hypothesis using 300 simulations based on the expected background: we computed the median, the $68 \%$, and the $95 \%$ confidence intervals of the expected upper limits on $\langle\sigma v\rangle$ for each DM mass. In the right panel of Fig. 3, we show a comparison of our constraints with limits obtained by other searches for gamma-ray line-like excesses. In case an On/Off region subtraction were performed to obtain the DM component as the residual, sensitivity would be lost for the core profile, due to 
the shallow gradient of the signal between the ON and OFF regions. Thanks to the sliding window technique, our analysis for a core profile suffers from less sensitivity degradation. Even with a conservative core profile [6], our limits are competitive with one of the most stringent limits derived from observations of dwarf spheroidal galaxies [11]. Assuming a cuspy Einasto profile, as also used in [5], our preliminary analysis on 204 hours of observation time provides limits on line-like DM annihilation in the DM mass range between $10 \mathrm{TeV}$ and $43 \mathrm{TeV}$ compatible with previous results based on 254 hours of observation time by H.E.S.S.[5].
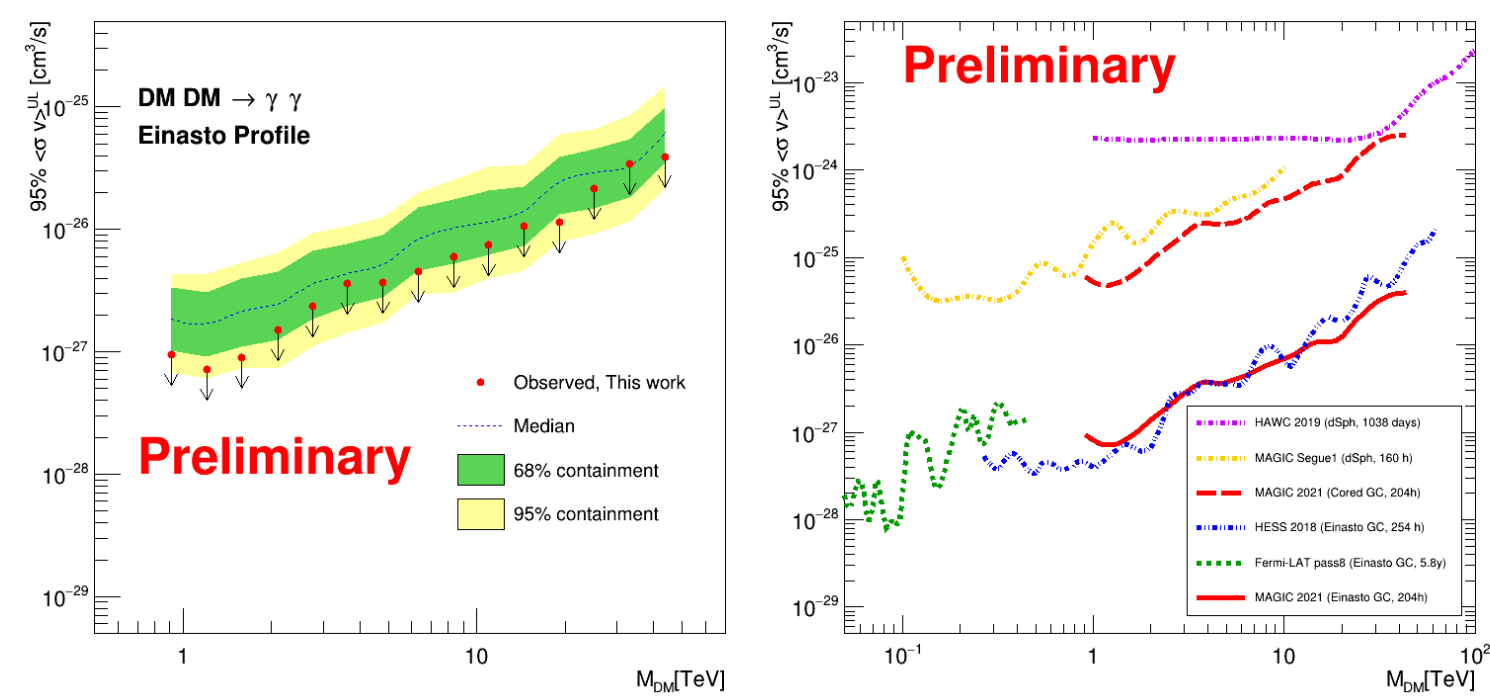

Figure 3: (Left) : 95\% C.L. upper limits on the annihilation cross section $\langle\sigma v\rangle$ assuming the Einasto profile. Observed limits (red dots with arrows) and the median of expected limits (black line) with the $68 \%$ and $95 \%$ containment bands with the null hypothesis are plotted together. (Right) : Comparison with results from other experiments. Observed limits on the annihilation cross section $\langle\sigma v\rangle$ (red solid line.) Also, limits by MAGIC for dwarf galaxy (orange dashed line, [11]), Fermi-LAT (green dashed line, [15]), H.E.S.S in 2018 (blue dashed line, [5]), and HAWC in 2019 (purple dashed line, [16]) are shown.

\section{Summary}

In this contribution, we have reported the upper limits on the DM annihilation cross section $\langle\sigma v\rangle$ obtained with 204 hours of data taken with the MAGIC telescopes observing the Galactic Centre. In particular, we have shown the potential for searches for a line-like gamma-ray signal with large zenith angle observations, which boost the sensitivity at high energies beyond several $\mathrm{TeV}$. Also, thanks to the sliding window technique, we could perform simultaneously the analysis with cuspy and core profiles with a competitive sensitivity. This clearly shows that large zenith angle observations will moreover provide an essential tool for the search of heavy DM signatures with the future Cherenkov Telescope Array. 


\section{Acknowledgements}

We acknowledge the support from the agencies and organizations listed under: https://magic.mpp.mpg.de/acknowledgments_ICRC2021

\section{References}

[1] Planck Collaboration, A\&A 594, A1 (2016), 383 arXiv:1502.01582 [astro-ph.CO].

[2] J. K. Gaskins, Contemp. phys. 57 (2016) 496-525 [arXiv:1604.00014]

[3] J. Hisano, S. Matsumoto, and M. M. Nojiri, 421 Phys. Rev. Lett. 92, 031303 (2004), arXiv:hepph/0307216 [hep-ph]

[4] E. Retana-Montenegro et al., A\&A 540, A70 (2012), arXiv:1202.5242 [astro-ph.CO].

[5] H.E.S.S. Collaboration, Phys. Rev. Lett. 120, 201101 (2018), arXiv:1805.05741 [astro-ph.HE].

[6] P. J. McMillan, MNRAS 465, 76 (2017), arXiv:1608.00971 [astro-ph.GA]

[7] MAGIC Collaboration, Astropart. Phys., 76-94 (2016), arXiv:1409.5594 [astro-ph.IM]

[8] MAGIC Collaboration, A\&A 642, A190 (2020), arXiv:2006.00623 [astro-ph.HE].

[9] R. Zanin, ICRC 2013 proceeding (2013) http://inspirehep.net/record/1412925/files/icrc20130773.pdf

[10] C. Weniger, JCAP. 2012, 007 (2012), arXiv:1204.2797 [hep-ph]

[11] MAGIC Collaboration, JCAP. 2014, 008 (2014), arXiv:1312.1535 [hep-ph]

[12] J. Rico et al., DOI 10.5281/zenodo.3967385

[13] MAGIC Collaboration, JCAP 2018, 009 (2018), arXiv:1712.03095 [astro-ph.HE]

[14] D. Ninci, T. Inada, J. Rico, et al., ICRC 2019 proceeding (2019) arXiv:1909.00222 [astroph.HE]

[15] Fermi LAT Collaboration, Phys. Rev. D 91, 122002 (2015), arXiv:1506.00013 [astro-ph.HE]

[16] HAWC Collaboration, Phys. Rev. D 101, 103001 (2020), arXiv:1912.05632 [astro-ph.HE]

\section{The MAGIC Collaboration}

V. A. Acciari ${ }^{1}$, S. Ansoldi ${ }^{2,41}$, L. A. Antonelli ${ }^{3}$, A. Arbet Engels $^{4}$, M. Artero ${ }^{5}$, K. Asano $^{6}$, D. Baack ${ }^{7}$, A. Babić ${ }^{8}$, A. Baquero ${ }^{9}$, U. Barres de Almeida ${ }^{10}$, J. A. Barrio ${ }^{9}$, I. Batković ${ }^{11}$, J. Becerra González ${ }^{1}$, W. Bednarek ${ }^{12}$, L. Bellizzi ${ }^{13}$, E. Bernardini ${ }^{14}$, M. Bernardos ${ }^{11}$, A. Berti ${ }^{15}$, J. Besenrieder ${ }^{15}$, W. Bhattacharyya ${ }^{14}$, C. Bigongiari ${ }^{3}$, A. Biland ${ }^{4}$, O. Blanch ${ }^{5}$, H. Bökenkamp ${ }^{7}$, G. Bonnoli ${ }^{16}$, Ž. Bošnjak ${ }^{8}$, G. Busetto ${ }^{11}$, R. Carosi ${ }^{17}$, G. Ceribella ${ }^{15}$, M. Cerruti ${ }^{18}$, Y. Chai ${ }^{15}$, A. Chilingarian ${ }^{19}$, S. Cikota ${ }^{8}$, S. M. Colak ${ }^{5}$, E. Colombo ${ }^{1}$, J. L. Contreras ${ }^{9}$, J. Cortina ${ }^{20}$, S. Covino ${ }^{3}$, G. D’Amico ${ }^{15,42}$, V. D’Elia ${ }^{3}$, P. Da Vela ${ }^{17,43}$, F. Dazzi ${ }^{3}$, A. De Angelis ${ }^{11}$, B. De Lotto ${ }^{2}$, M. Delfino ${ }^{5,44}$, J. Delgado ${ }^{5,44}$, C. Delgado Mendez $^{20}$, D. Depaoli' ${ }^{21}$, F. Di Pierro ${ }^{21}$, L. Di Venere ${ }^{22}$, E. Do Souto Espiñeira ${ }^{5}$, D. Dominis Prester ${ }^{23}$, A. Donini ${ }^{2}$, D. Dorner ${ }^{24}$, M. Doro ${ }^{11}$, D. Elsaesser ${ }^{7}$, V. Fallah Ramazani ${ }^{25,45}$, A. Fattorini ${ }^{7}$, M. V. Fonseca ${ }^{9}$, 
L. Font ${ }^{26}$, C. Fruck ${ }^{15}$, S. Fukami ${ }^{6}$, Y. Fukazawa ${ }^{27}$, R. J. García López ${ }^{1}$, M. Garczarczyk ${ }^{14}$, S. Gasparyan ${ }^{28}$, M. Gaug ${ }^{26}$, N. Giglietto ${ }^{22}$, F. Giordano ${ }^{22}$, P. Gliwny ${ }^{12}$, N. Godinović ${ }^{29}$, J. G. Green ${ }^{3}$, D. Green ${ }^{15}$, D. Hadasch ${ }^{6}$, A. Hahn ${ }^{15}$, L. Heckmann ${ }^{15}$, J. Herrera ${ }^{1}$,

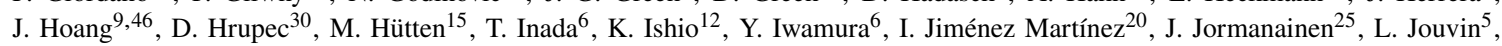
M. Karjalainen ${ }^{1}$, D. Kerszberg 5 , Y. Kobayashi ${ }^{6}$, H. Kubo ${ }^{31}$, J. Kushida ${ }^{32}$, A. Lamastra ${ }^{3}$, D. Lelas ${ }^{29}$, F. Leone ${ }^{3}$, E. Lindfors ${ }^{25}$, L. Linhoff ${ }^{7}$, S. Lombardi ${ }^{3}$, F. Longo ${ }^{2,47}$, R. López-Coto ${ }^{11}$, M. López-Moya ${ }^{9}$, A. López-Oramas ${ }^{1}$, S. Loporchio ${ }^{22}$, B. Machado de Oliveira Fraga $^{10}$, C. Maggio ${ }^{26}$, P. Majumdar ${ }^{33}$, M. Makariev ${ }^{34}$, M. Mallamaci ${ }^{11}$, G. Maneva ${ }^{34}$, M. Manganaro ${ }^{23}$, K. Mannheim $^{24}$, L. Maraschi ${ }^{3}$, M. Mariotti ${ }^{11}$, M. Martínez ${ }^{5}$, D. Mazin ${ }^{6,15}$, S. Menchiari ${ }^{13}$, S. Mender ${ }^{7}$, S. Mićanović2 ${ }^{23}$, D. Miceli ${ }^{2,49}$, T. Miener ${ }^{9}$, J. M. Miranda ${ }^{13}$, R. Mirzoyan ${ }^{15}$, E. Molina ${ }^{18}$, A. Moralejo ${ }^{5}$, D. Morcuende ${ }^{9}$, V. Moreno ${ }^{26}$, E. Moretti ${ }^{5}$, T. Nakamori ${ }^{35}$, L. Nava ${ }^{3}$, V. Neustroev ${ }^{36}$, C. Nigro ${ }^{5}$, K. Nilsson ${ }^{25}$, K. Nishijima ${ }^{32}$, K. Noda $^{6}$, S. Nozaki ${ }^{31}$, Y. Ohtani' ${ }^{6}$, T. Oka ${ }^{31}$, J. Otero-Santos ${ }^{1}$, S. Paiano ${ }^{3}$, M. Palatiello ${ }^{2}$, D. Paneque ${ }^{15}$, R. Paoletti ${ }^{13}$, J. M. Paredes ${ }^{18}$, L. Pavletić ${ }^{23}$, P. Peñil ${ }^{9}$, M. Persic ${ }^{2,50}$, M. Pihet ${ }^{15}$, P. G. Prada Moroni ${ }^{17}$, E. Prandini ${ }^{11}$, C. Priyadarshi ${ }^{5}$, I. Puljak ${ }^{29}$, W. Rhode ${ }^{7}$, M. Ribó ${ }^{18}$, J. Rico ${ }^{5}$, C. Righi ${ }^{3}$, A. Rugliancich ${ }^{17}$, N. Sahakyan ${ }^{28}$, T. Saito ${ }^{6}$, S. Sakurai ${ }^{6}$, K. Satalecka ${ }^{14}$, F. G. Saturni ${ }^{3}$, B. Schleicher ${ }^{24}$, K. Schmidt $^{7}$, T. Schweizer ${ }^{15}$, J. Sitarek ${ }^{12}$, I. Šnidaric ${ }^{37}$, D. Sobczynska ${ }^{12}$, A. Spolon ${ }^{11}$, A. Stamerra ${ }^{3}$, J. Strišković ${ }^{30}$, D. Strom ${ }^{15}$, M. Strzys ${ }^{6}$, Y. Suda ${ }^{27}$, T. Suric ${ }^{37}$, M. Takahashi ${ }^{6}$, R. Takeishi ${ }^{6}$, F. Tavecchio ${ }^{3}$, P. Temnikov ${ }^{34}$, T. Terzić ${ }^{23}$, M. Teshima ${ }^{15,6}$, L. Tosti $^{38}$, S. Truzzi ${ }^{13}$, A. Tutone ${ }^{3}$, S. Ubach ${ }^{26}$, J. van Scherpenberg ${ }^{15}$, G. Vanzo $^{1}$, M. Vazquez Acosta ${ }^{1}$, S. Ventura ${ }^{13}$, V. Verguilov ${ }^{34}$, C. F. Vigorito ${ }^{21}$, V. Vitale ${ }^{39}$, I. Vovk $^{6}$, M. Will ${ }^{15}$, C. Wunderlich ${ }^{13}$, T. $_{\text {Yamamoto }}{ }^{40}$, and D. Zaric ${ }^{29}$

${ }^{1}$ Instituto de Astrofísica de Canarias and Dpto. de Astrofísica, Universidad de La Laguna, E-38200, La Laguna, Tenerife, Spain ${ }^{2}$ Università di Udine and INFN Trieste, I-33100 Udine, Italy ${ }^{3}$ National Institute for Astrophysics (INAF), I-00136 Rome, Italy ${ }^{4}$ ETH Zürich, CH-8093 Zürich, Switzerland ${ }^{5}$ Institut de Física d'Altes Energies (IFAE), The Barcelona Institute of Science and Technology (BIST), E-08193 Bellaterra (Barcelona), Spain ${ }^{6}$ Japanese MAGIC Group: Institute for Cosmic Ray Research (ICRR), The University of Tokyo, Kashiwa, 277-8582 Chiba, Japan ${ }^{7}$ Technische Universität Dortmund, D-44221 Dortmund, Germany ${ }^{8}$ Croatian MAGIC Group: University of Zagreb, Faculty of Electrical Engineering and Computing (FER), 10000 Zagreb, Croatia ${ }^{9}$ IPARCOS Institute and EMFTEL Department, Universidad Complutense de Madrid, E-28040 Madrid, Spain ${ }^{10}$ Centro Brasileiro de Pesquisas Físicas (CBPF), 22290-180 URCA, Rio de Janeiro (RJ), Brazil ${ }^{11}$ Università di Padova and INFN, I-35131 Padova, Italy ${ }^{12}$ University of Lodz, Faculty of Physics and Applied Informatics, Department of Astrophysics, 90-236 Lodz, Poland ${ }^{13}$ Università di Siena and INFN Pisa, I-53100 Siena, Italy ${ }^{14}$ Deutsches Elektronen-Synchrotron (DESY), D-15738 Zeuthen, Germany ${ }^{15}$ Max-Planck-Institut für Physik, D-80805 München, Germany ${ }^{16}$ Instituto de Astrofísica de Andalucía-CSIC, Glorieta de la Astronomía s/n, 18008, Granada, Spain ${ }^{17}$ Università di Pisa and INFN Pisa, I-56126 Pisa, Italy ${ }^{18}$ Universitat de Barcelona, ICCUB, IEEC-UB, E-08028 Barcelona, Spain ${ }^{19}$ Armenian MAGIC Group: A. Alikhanyan National Science Laboratory, 0036 Yerevan, Armenia ${ }^{20}$ Centro de Investigaciones Energéticas, Medioambientales y Tecnológicas, E-28040 Madrid, Spain ${ }^{21}$ INFN MAGIC Group: INFN Sezione di Torino and Università degli Studi di Torino, I-10125 Torino, Italy 22 INFN MAGIC Group: INFN Sezione di Bari and Dipartimento Interateneo di Fisica dell'Università e del Politecnico di Bari, I-70125 Bari, Italy ${ }^{23}$ Croatian MAGIC Group: University of Rijeka, Department of Physics, 51000 Rijeka, Croatia ${ }^{24}$ Universität Würzburg, D-97074 Würzburg, Germany ${ }^{25}$ Finnish MAGIC Group: Finnish Centre for Astronomy with ESO, University of Turku, FI-20014 Turku, Finland ${ }^{26}$ Departament de Física, and CERES-IEEC, Universitat Autònoma de Barcelona, E-08193 Bellaterra, Spain ${ }^{27}$ Japanese MAGIC Group: Physics Program, Graduate School of Advanced Science and Engineering, Hiroshima University, 739-8526 Hiroshima, Japan ${ }^{28}$ Armenian MAGIC Group: ICRANet-Armenia at NAS RA, 0019 Yerevan, Armenia ${ }^{29}$ Croatian MAGIC Group: University of Split, Faculty of Electrical Engineering, Mechanical Engineering and Naval Architecture (FESB), 21000 Split, Croatia ${ }^{30}$ Croatian MAGIC Group: Josip Juraj Strossmayer University of Osijek, Department of Physics, 31000 Osijek, Croatia ${ }^{31}$ Japanese MAGIC Group: Department of Physics, Kyoto University, 606-8502 Kyoto, Japan ${ }^{32}$ Japanese MAGIC Group: Department of Physics, Tokai University, Hiratsuka, 259-1292 Kanagawa, Japan ${ }^{33}$ Saha Institute of Nuclear Physics, HBNI, 1/AF Bidhannagar, Salt Lake, Sector-1, Kolkata 700064, India ${ }^{34}$ Inst. for Nucl. Research and Nucl. Energy, Bulgarian Academy of Sciences, BG-1784 Sofia, Bulgaria ${ }^{35}$ Japanese MAGIC Group: Department of Physics, Yamagata University, Yamagata 990-8560, Japan ${ }^{36}$ Finnish MAGIC Group: Astronomy Research Unit, University of Oulu, FI-90014 Oulu, Finland ${ }^{37}$ Croatian MAGIC Group: Ruđer Bošković Institute, 10000 Zagreb, Croatia ${ }^{38}$ INFN MAGIC Group: INFN Sezione di Perugia, I-06123 Perugia, Italy ${ }^{39}$ INFN MAGIC Group: INFN Roma Tor Vergata, I-00133 Roma, Italy ${ }^{40}$ Japanese MAGIC Group: Department of Physics, Konan University, Kobe, Hyogo 6588501, Japan ${ }^{41}$ also at International Center for Relativistic Astrophysics (ICRA), Rome, Italy ${ }^{42}$ now at Department for Physics and Technology, University of Bergen, NO-5020, Norway ${ }^{43}$ now at University of Innsbruck ${ }^{44}$ also at Port d'Informació Científica (PIC), E-08193 Bellaterra (Barcelona), Spain ${ }^{45}$ now at Ruhr-Universität Bochum, Fakultät für Physik und Astronomie, Astronomisches Institut (AIRUB), 44801 Bochum, Germany ${ }^{46}$ now at Department of Astronomy, University of California Berkeley, Berkeley CA $94720{ }^{47}$ also at Dipartimento di Fisica, Università di Trieste, I-34127 Trieste, Italy ${ }^{49}$ now at Laboratoire d'Annecy de Physique des Particules (LAPP), CNRS-IN2P3, 74941 Annecy Cedex, France ${ }^{50}$ also at INAF Trieste and Dept. of Physics and Astronomy, University of Bologna, Bologna, Italy 